\title{
Dairy animal health - turning problems into opportunities
}

\author{
J Eric Hillerton
}

Massey University, Palmerston North 4442, New Zealand

As one of the Executive Editorial Team for the Journal of Dairy Research, I am pleased to provide this Editorial for the May issue. Every dairy farmer knows that only healthy animals are fully productive, both for quantity and quality of milk. Detecting health abnormalities is a challenge to those farming large herds; often the farmer is looking for the 'needle in the haystack'. Fortunately, new detection and sensor technologies are being introduced that are already particularly relevant for dairy cattle farmers and may find wider application in the future as interest in other dairy species grows. One possible route to application of these technologies is described in this issue (Caja et al. 2016). Maintaining health includes managing disease and preventing new infections, which together incur costs that may be significant in the farm budget. With a major depression in world milk prices, farmers are facing financial challenges to their very existence and most will look to cut their animal health budget despite risking any of many potential disease outbreaks.

Mastitis is the most expensive of all diseases affecting dairy herds of all species and all countries. Lameness and reproductive tract problems complete the most important three in all dairy systems. Mastitis is arguably the disease complex against which most progress has been made, probably now being two thirds lower in dairy cows than before control programmes were introduced. For 50 years dairy animals have benefited from the use of antimicrobials to cure and prevent the bacterial infections that result in mastitis. Now the very access to antimicrobial products used for therapy, and especially for prophylaxis, is threatened by responses, political and trade, to the rapid growth of antimicrobial resistance. This may be a huge problem in dealing with bacteria affecting human health but, so far, evidence of a problem at the production level for dairy animals is scant. However, pressure is growing, and will not dissipate, to reduce veterinary use of antimicrobials.

Bacterial infections are the predominant cause of mammary gland inflammation and receive almost exclusive attention in cows. Bacterial infections are equally important for other dairy production industries; they may be rarely reported for other animals but receive much warranted attention when affecting mothers who are nursing human babies. I am far from the first researcher to be motivated in my work by such a problem at home, but human mastitis research lags far behind bovine. Other microorganisms may cause disease or at least may be shed and infection spread from their presence in milk. Foot and Mouth Disease can be readily diagnosed from milk samples as can Bovine Viral Diarrhoea; caprine arthritis is widely distributed, and HIV transmission to babies via breastmilk during sub-clinical mastitis is a problem in parts of sub Saharan Africa.

Every set of problems bring opportunities. To extend the useful life of antimicrobials in managing animal health and in postponing the 'post antibiotic era', as imagined by the World Health Organisation, dairy scientists have much to do and all this work lies within the scope of the Journal of Dairy Research. Farmers and veterinarians urgently need to make more astute use of antimicrobial treatments, treating only affected animals and those most at risk. In the coming few years they will need alternative and new technologies, spanning from better diagnostics to means of enhancing the immunological responses of the animals at risk. Better still may be to incorporate into breeding programmes even greater genetic resistance to the more common diseases. Many other genetic developments will be welcome, as an example the February issue included a review on gene editing (Whitelaw et al. 2016).

On a technical note, this issue of the Journal introduces one of the important changes being made, the publication of Research Communications - short articles reporting good science. The first, on goats and opening this issue's Original Article contents, illustrates the multi-species scope of the Journal. The Research Communication that concludes this issue, on possible residues in milk powder, exemplifies the range of topics that are relevant to the Journal. Its placement is entirety fortuitous, and the two together reinforce the point that Research Communications will be treated equally with full length papers and never as afterthoughts. The criteria for publications, be they papers or communications, are scientific quality and relevance to the scope of the Journal.

\section{References}

Caja G, Castro-Costa A \& Knight CH 2016 Engineering to support wellbeing of dairy animals. Journal of Dairy Research 83 136-147

Whitelaw CBA, Joshi A, Kumar S, Lillico SG \& Proudfoot C 2016 Genetically engineering milk. Journal of Dairy Research 83 3-11

For correspondence; e-mail: hillerton@netsmart.net.nz 